\title{
JC virus regulatory region rearrangements in the brain of a long surviving patient with progressive multifocal leukoencephalopathy
}

\author{
Y Yogo, T Matsushima-Ohno, T Hayashi, C Sugimoto, M Sakurai, I Kanazawa
}

\begin{abstract}
JC virus (JCV) infection of oligodendrocytes causes demyelination in brains of patients with with progressive multifocal leukoencephalopathy (PML). Expansion of demyelination throughout the brain is not fully understood. The opportunity was taken to investigate the postmortem brain of a long surviving patient with PML for whom diagnosis was made 4 years before death based on pathological and virological findings of a brain biopsy. Four distinct regulatory sequences in the JCV genome were detected (designated as JW-1 to 4 ) from various regions of the necropsied brain. All regulatory sequences were rearranged forms that could be produced from the archetype by deletions and duplications. JW-1 and 2 shared some structural features not present in JW-3 and 4 and vice versa. JW-1 was distributed throughout the brain, whereas $\mathrm{JW}-2,3$, and 4 were restricted to only part of the brain. JW-1 and 2 had been detected in the initial brain biopsy 4 years earlier. These findings suggested that brain lesions in advanced stages were generated not only by expansion of the original variant (JW-1) of JCV but also by delayed growth of two other variants (JW-3 and 4$)$.
\end{abstract}

(F Neurol Neurosurg Psychiatry 2001;71:397-400)

Keywords: progressive multifocal leukoencephalopathy; JC virus; DNA sequences

In the CNS with progressive multifocal leukoencephalopathy (PML), JC virus (JCV) replication in oligodendrocytes results in the loss of myelin, leading to severe deterioration of brain function. ${ }^{12}$ It is not clear how JCV invades the CNS or how the lesions caused by JCV expand in the CNS. Focal lesions of demyelination initially develop in one region of the brain, but in the course of PML progression, they may also be found in other areas. The lesions generated during advanced stages of the disease may be produced by migration of JCV from the original area of demyelination. Alternatively, demyelination during advanced stages may be due to a JCV of a different origin.
Brain JCV DNAs in the patients with PML carry various regulatory regions (PML-type regulatory regions) produced by rearrangements (deletions and duplications) of a single archetype structure. ${ }^{3-7}$ Because rearrangements in the JCV regulatory region occur randomly, it should be possible to distinguish individual regulatory regions. It was recently suggested that variants in JCV regulatory regions may help trace the pathway of JCV from the site of initial infection to the target oligodendrocytes. ${ }^{8}$ We hypothesised that JCV regulatory rearrangements might be helpful for tracing the development of demyelinating lesions in the brain.

If we detect identical or related regulatory regions of JCV in different parts of the brain, we may speculate that demyelination in these parts of the brain was caused by JCVs of the same origin. If we detect unrelated regulatory regions in different parts of the brain, demyelination in these areas was likely caused by JCVs of different origins. We report here that JCV regulatory region rearrangements were used successfully to follow the expansion of brain lesions in a long surviving patient with PML. ${ }^{9}$

\section{Patient presentation}

The case report was documented at the time of diagnosis. ${ }^{9}$ Briefly, the patient was a 34 year old man who experienced an epileptic attack in September 1992, but he had apparently normal neurological findings. He was admitted to hospital in January 1994 because of slowly progressing dementia. The underlying generalised disease in this patient was diagnosed as Wiscott-Aldrich syndrome. Brain MRI showed multiple tiny spots of Gd-DTPA enhanced lesions on a T1 weighted image. Pathological findings for a brain biopsy were patchy, demyelinated, vascularised lesions infiltrated by many eosinophils. In July 1994, a diagnosis of PML was made on the basis of brain biopsy findings, including detection of JCV DNA by polymerase chain reaction (PCR) and in situ hybridisation. Multiple diffuse spotty lesions were found initially in the frontal lobe, but they gradually appeared in other parts of the cerebrum, cerebellar white matter, and brain stem. The patient survived in a vegetative state for 4 years after the diagnosis was made, and he 
died from central cardiorespiratory failure on 16 October 1998. Necropsy findings showed severe brain atrophy and diffuse demyelination of the white matter of the cerebrum, cerebellum, and spinal cord.

\section{Analysis of JCV regulatory sequences}

The JCV regulatory region was amplified from genomic DNA with the B1/B4 primer pair. B1 was described previously ${ }^{10}$; B4 was $5^{\prime}$ GCCTGCAGTTACTTACCTATGTAGCTTT 3', which corresponds to nucleotides (nt) 500 to
481 of the JCV (Mad-1) genome (fig $1 \mathrm{~A}$ ). ${ }^{1}$ The underlined octanucleotide was added to create a Pst I cleavage site. Amplification was performed as described previously. ${ }^{10}$ Purified PCR products were digested with a combination of HindIII and PstI, which excises a fragment containing the regulatory region (fig $1 \mathrm{~A})$. The recovered DNA was cloned into pBluesript II SK (+) (Stratagene, La Jolla, CA, USA). Representative recombinant clones were sequenced with an autosequencer.

A

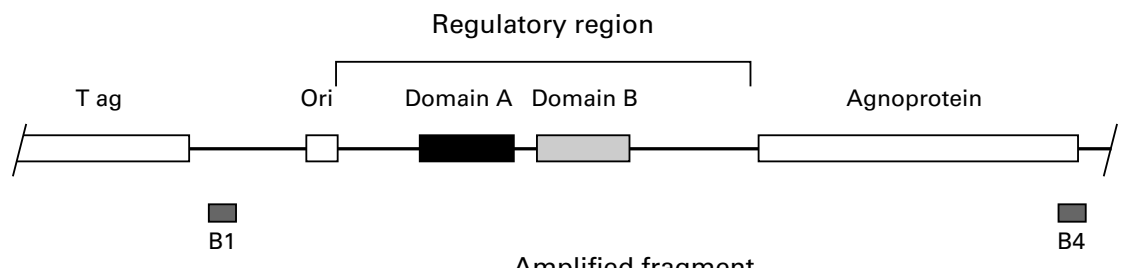

Amplified fragment

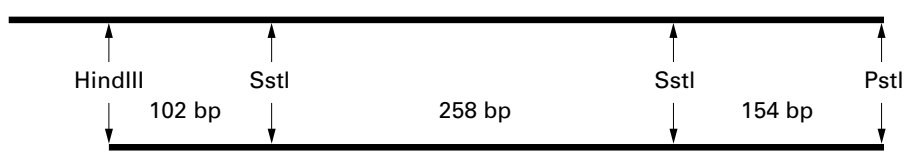

Cloned fragment

B

Archtype

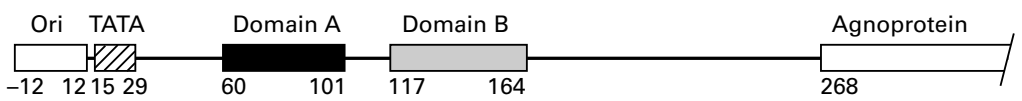

JW-1
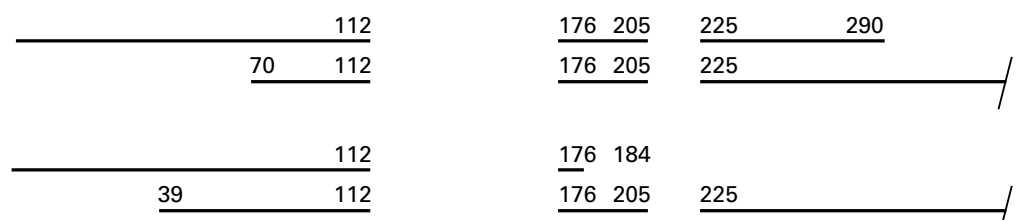

JW-2

39

112

Intermediate-1

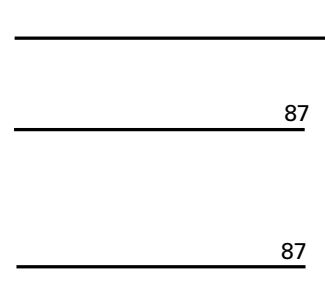

112

JW-3

JW-4

87

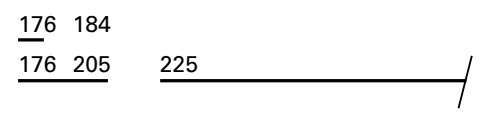

$\underline{176 \quad 205}$

$\underline{225}$

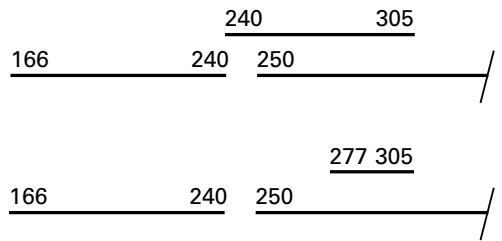

Intermediate-2

305

166

$240 \quad 250$

Figure 1 (A) Sites of primers used for PCR. A portion of the $7 C V$ genome including the archetype regulatory region is shown schematically at the top. T antigen gene (T ag), origin of replication (Ori), and agnoprotein gene are indicated. ${ }^{1}$ Domain A indicates the sequence duplicated in many PML derived FCV isolates, and domain B indicates that the sequence deleted in many PML derived $\mathcal{F} C V$ isolates. ${ }^{4}$ Below the partial $\mathcal{F} C V$ genome, the fragment that was amplified by PCR and the subfragment that was cloned into pBluesript II SK (+) and subsequently sequenced are shown. HindIII, SstI, and PstI cleavage sites are indicated on the amplified and cloned fragments. In addition, the sizes of subfragments generated from clones with the archetype regulatory region are shown. It should be noted that the sizes of subfragments generated from the clones with PML-type regulatory regions varied depending on the sequence rearrangements in the regulatory region (table 1). (B) Diagrammatic representation of the detected $\mathcal{F C V}$ regulatory sequences. The structure of the archetypal regulatory region is shown schematically at the top. Symbols are as in $(A)$. The $\mathcal{F} C V$ regulatory regions recovered from brain tissue ( $(W-1$ to 4) and hypothetical intermediates ( 1 and 2) are shown with deletions relative to the archetype indicated as gaps. Reading from left to right, when a repeat was encountered, the linear representation is displaced to the line below and to a position corresponding to the sequence of the archetype. Numbers below each box and line are nucleotide numbers indicating end locations (nucleotide numbering system is similar to that of Frisque et al. ${ }^{11}$ ). 
Table 1 Distribution of various $\mathcal{F C V}$ regulatory regions in the brain *

\begin{tabular}{|c|c|c|c|c|c|c|}
\hline \multirow[b]{2}{*}{ Part of the brain } & \multirow{2}{*}{$\begin{array}{l}\text { Total No } \\
\text { of clones }\end{array}$} & \multicolumn{5}{|c|}{ Incidence of regulatory region (\%) } \\
\hline & & $\mathcal{F} W-1$ & $\mathcal{f} W-2$ & $\mathcal{f} W-3$ & $\mathcal{F W}-4$ & Others \\
\hline Frontal lobe & 32 & 91 & 9 & 0 & 0 & 0 \\
\hline Parietal lobe & 32 & 91 & 6 & 0 & 0 & 3 \\
\hline Temporal lobe & 31 & 7 & 93 & 0 & 0 & 0 \\
\hline Occipital lobe & 26 & 70 & 0 & 4 & 27 & 0 \\
\hline Cerebellum & 34 & 50 & 0 & 38 & 11 & 0 \\
\hline Pons & 26 & 96 & 0 & 4 & 0 & 0 \\
\hline Frontal lobe & 31 & 77 & 13 & 0 & 0 & $10 \ddagger$ \\
\hline
\end{tabular}

*Using primers $\mathrm{B} 1$ and $\mathrm{B} 4$, a portion of the JCV genome including the regulatory region and the agnoprotein gene (see fig 1A) was amplified from various parts of the brain. The amplified fragments were then cloned into pBluesript II SK (+) (Stratagene, La Jolla, CA, USA), and 26 to 32 recombinant clones were digested with a combination of HindIII, PstI, and SstI. According to the generated fragment patterns, clones were classified as JW-1, 2, 3, or 4. The sizes of subfragments generated from each regulatory region were as follows (shown in base pairs): JW-1 $(176,154,139$, 102), JW-2 (176, 154, 102, 83), JW-3 (237, 154, 102), and JW-4 $(200,154,102)$.

†Brain biopsy obtained 4 years before the patient's death.

$\ddagger$ Regulatory regions produced from JW-1 by further amplifications

We detected four distinct JCV regulatory regions, designated JW-1, 2, 3, and 4, from various regions of the brain (fig $1 \mathrm{~B}$ ). All regulatory regions were PML-type regulatory regions and could be grouped into two classes, JW-1/2 and JW-3/4. JW-1/2 lacked two segments, one spanning nt 113 to 175 and the other spanning nt 206 to 224 . JW-3/4 lacked the segment spanning nt 241 to 249 . In addition, the end points of the duplicated segments in JW-3/4 were the same (nt 166 and 305). These structural features indicated that JW-1/2 and JW-3/4 developed through different intermediates (intermediates 1 and 2 shown in fig 1). In other words, JW-1/2 and JW-3/4 were generated independently from the archetype sequence. In addition, we found that 610 base pair sequences (VT-intergenic sequences) of the JCV genome, ${ }^{12}$ usually used to distinguish JCV strains, differ between JW-1/2 and JW-3/4 by two nucleotide mismatches (data not shown), suggesting that JW-1/2 and JW-3/4 were produced from different archetype JCV strains.

Fragments of JCV DNA were amplified by PCR from various regions of the brain. Recombinant clones of the amplified fragments were classified according to restriction enzyme analysis (table 1). JW-1 was distributed widely throughout the brain; its incidence varied from greater than $90 \%$ (frontal and parietal lobes and pons) to less than $10 \%$ (temporal lobe). JW-2 was restricted to the cerebrum with the highest incidence (93\%) in the temporal lobe. JW-3 was found only in the cerebellum, occipital lobe, and pons with a higher incidence $(38 \%)$ in the cerebellum. JW-4 was found only in the occipital lobe and cerebellum at relatively low incidences $(11 \%$ and $27 \%$, respectively).

One major sequence (JW-1) and one minor sequence (JW-2) were detected in the biopsy of brain tissue obtained 4 years before the patient's death (table 1). ${ }^{9}$ This pattern was similar to that in the same region of the necropsied brain (table 1 ).

\section{Discussion}

We studied the postmortem brain of a long surviving patient with PML for whom diagnosis was made 4 years before the patient's death.
The JCV regulatory sequence data combined with the neuroradiological findings provide new insights into the expansion of brain lesions.

We detected JW-1 and 2 in a brain biopsy obtained from the frontal lobe 4 years before death. ${ }^{9}$ The detection of JW- 1 and 2 in the same region of the brain where earlier lesions were shown by neuroradiological observations suggests that JCVs with the JW- 1 and 2 regulatory regions cause earlier lesions in the CNS. Four years later, we had the opportunity to examine the necropsy brain in detail. In addition to JW-1 and 2, other regulatory sequences (JW-3 and 4) were detected. The marked structural differences between JW-1/2 and JW-3/4 (fig 1) excluded the possibility that JW-3 and 4 were derived from JW-1 or JW-2. The topological distributions of the four rearranged regulatory sequences (table 1) implies that the late lesions in the CNS were generated by expansion of a JCV variant with JW-1 as well as delayed growth of JCVs with other rearrangements. However, it is unclear why the other early variant with JW-2 did not expand in the CNS.

The current evidence suggests that JCV is not present in the CNS latently but appears there only after reactivation. ${ }^{13}$ Thus, the scenario proposed by Major et al seems most likely. ${ }^{14}$ Briefly, after primary infection, JCV persists in peripheral lymphocytes, and when the host becomes immunologically impaired, the latent virus begins to grow efficiently, resulting in the expansion of JCV infection. Some of the infected lymphocytes invade the CNS, followed by the infection of oligodendrocytes and astrocytes.

The rearrangement of regulatory regions that generated JW-1, 2, 3, and 4 involved two steps: from the archetype to intermediates and from intermediates to fully rearranged structures. The possible sites where these rearrangements occurred were peripheral blood lymphocytes and the CNS. The occurrence of related regulatory regions (JW-1/2 or JW-3/4) in the same or neighbouring regions of the CNS (table 1) suggests that the change from intermediates to PML-type regulatory regions occurred in the CNS, although the initial change from the archetype to intermediates may have occurred in peripheral blood lymphocytes.

In summary, we found that multiple JCV variants with different regulatory region structures occurred in the CNS in the present patient. The JCV regulatory sequence data, combined with the neuroradiological findings, provided a new insight into the expansion of brain lesions. As the present patient was rather unique, it remains to investigate other patients with PML using the approach used in this study.

1 ZuRhein GM. Association of papova-virions with a human demyelinating disease (progressive multifocal leukoencephalopathy). Prog Med Virol 1969;11:185-247.

2 Padgett BL, Walker DL, ZuRhein GM, et al. Cultivation of papova-like virus from human brain with progressive multifocal leukoencephalopathy. Lancet 1971;i:1257-60. 
3 Yogo Y, Kitamura T, Sugimoto C, et al. Isolation of a possible archetypal JC virus DNA sequence from nonimmunocompromised individuals. F Virol 1990;64:3139-43.

4 Iida T, Kitamura T, Guo J, et al. Origin of JC virus variants associated with progressive multifocal leukoencephalopathy. Proc Natl Acad Sci USA 1993;90:5062-5.

5 Ault GS, Stoner GL. Human polyomavirus JC promoter enhancer rearrangement patterns from progressive multifocal leukoencephalopathy brain are unique derivatives of a single archetypal structure. F Gen Virol 1993;74:1499-507.

6 Agostini HT, Ryschkewitsch CF, Singer EJ, et al. JC virus regulatory region rearrangements and genotypes in pro-
gressive multifocal leukoencephalopathy: two independent aspects of virus variation. F Gen Virol 1997;78:659-64.

7 Yogo Y, Sugimoto C. The archetype concept and regulatory region rearrangement. In: Khalili K, Stoner, GL, eds. The human polyomaviruses $\mathcal{F} C, B K$, and SV40: molecular and clinical perspectives. New York: John Wiley, in press.

8 Jensen PN, Major EO Viral variant nucleotide sequences help expose leukocytic positioning in the JC virus pathway to the CNS. F Leukoc Biol 1999;65:428-38.
9 Matsushima T, Nakamura $\mathrm{K}$, Oka $\mathrm{T}$, et al. Unusual MRI and pathologic findings of progressive multifocal leukoencephalopathy complicating adult Wiskott-Aldrich syndrome. Neurology 1997;48:279-82.

10 Sugimoto C, Ito D, Tanaka K, et al. Amplification of JC virus regulatory DNA sequences from cerebrospinal fluid: diagnostic value for progressive multifocal leukoencephalopathy. Arch Virol 1998;143:249-62.

11 Frisque RJ, Bream GL, Cannella MT. Human polyomavirus JC virus genome. 7 Virol 1984;51:458-69.

12 Kunitake T, Kitamura T, Guo J, et al. Parent-to-child transmission is relatively common in the spread of the human polyomavirus JC virus. F Clin Microbiol 1995;33: 1448-51.

13 Berger JR, Major EO. Progressive multifocal leukoencephalopathy. Semin Neurol 1999;19:193-200.

14 Major EO, Amemiya K, Tornatore CS, et al. Pathogenesis and molecular biology of progressive multifocal leukoencephalopathy, the JC virus-induced demyelinating disease of the human brain. Clin Microbiol Rev 1992;5:49-73.

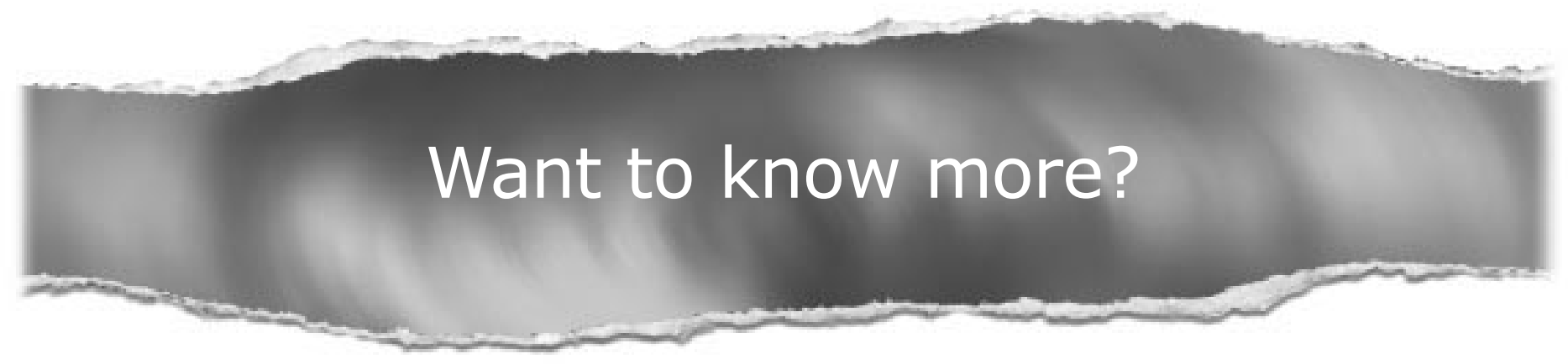

Data supplements

Limited space in printed journals means that interesting data and other material are often edited out of articles; however, limitless cyberspace means that we can include this information online.

Look out for additional tables, references, illustrations.

www.jnnp.com 